\title{
The chances of a spina bifida patient becoming continent/socially dry by conservative therapy
}

\author{
M Knoll, H Madersbacher \\ Department of Urology, University Hospital, Innsbruck, Austria.
}

One hundred and one patients with neurogenic lower urinary tract dysfunction due to myelomeningocele or sacral dysplasia, all older than 10 years of age, with a mean age of 15 years, and under regular urological control for many years were evaluated to discover if they had achieved urinary continence (dry day and night) or the status of socially dry (with dry intervals of more than 3 hours) with conservative means of treatment; or whether they remained incontinent or required operative procedures. The results were correlated with the urodynamic pattern of their detrusor-sphincter dysfunction and with the methods they used for bladder emptying. Special attention was paid to the effects of the various conservative treatment modalities nowadays available.

The chances of becoming continent or at least socially dry by conservative therapy are best for patients with sphincter hyperreflexia provided that (1) the detrusor was already hypo- or areflexic, or detrusor hyperreflexia could be successfully controlled, and (2) that regular bladder emptying was achieved by clean intermittent (self) catheterisation. Twenty-four out of 45 patients in this group fullfilled these criteria: 18 are continent, 6 socially dry, a success rate of $54 \%$. In patients with sphincter weakness the outlook is worse: in combination with detrusor hyperreflexia there is only a $16 \%$ chance, and in those with detrusor areflexia a $37 \%$ chance of becoming continent or socially dry, with the best results in wheelchair patients. Adequate urological care from birth or early childhood onwards, as is the case in the majority of our patients, is another factor which influences a good outcome in these children, and may explain our favourable results with conservative means of treatment. In suitable patients additional intravesical electrotherapy improved the results considerably.

An overall success rate of $40 \%$ indicates that it is worthwhile to try conservative therapy to control urinary incontinence in these patients before major surgery is undertaken.

Keywords: myelodyplasia; neurogenic urinary incontinence; conservative therapy.

\section{Introduction}

Neurogenic dysfunction of the lower urinary tract is characterised by two main symptoms: insufficient bladder emptying and involuntary escape of urine. The aims of treatment are preservation of kidney function and the control of urinary incontinence. Preservation of kidney function is surely of prime importance. However, the problem of incontinence also needs to be solved once the children grow older, in order to avoid medical, psychological and social problems.
Children, who are wet, smelly and uncomfortable rapidly become outcasts and, constantly soiled by their own excretions, live miserable lives. Neurogenic urinary incontinence is caused either by detrusor hyperreflexia or by sphincter weakness, but often by a combination of both (vide infra).

Urinary incontinence can be managed basically by conservative or by operative treatment. The aim of this paper is to discuss the chances of a spina bifida patient 
becoming continent or at least socially dry by conservative means of treatment.

\section{Conservative means of therapy}

Conservative means of therapy for neurogenic urinary incontinence basically comprise so called bladder training, pharmacotherapy, long term infection prophylaxis and intravesical electrotherapy.

Bladder training is based primarily on regular bladder emptying by the clock by whatever means, with low residual urine and physiological detrusor pressure. In this regard, bladder emptying by clean intermittent (self) catheterisation (CIC) is of great importance. The frequency of CIC depends on bladder capacity; the bladder is usually catheterised 4 times a day. Moreover, regular and controlled fluid intake helps to achieve this goal.

Pharmacotherapy is nowadays mainly focused on the relaxation of a hyperreflexive (spastic) bladder in order to control detrusor hyperreflexia as a cause of involuntary escape of urine. With the help of a new generation of smooth muscle relaxant medicaments with anticholinergic, direct spasmolytic, calcium-channel blocking and local anaesthetic properties, eg oxybutynine hydrochloride $\left(\right.$ Ditropan $^{\mathrm{R}}$, Dridase ${ }^{\mathrm{R}}$ ), Terodiline $\left(\right.$ Mictral $\left.^{R}\right)$, Trospium chloride (Spasmolyt $\left.{ }^{\mathrm{R}}\right)$, hyperreflexia of the detrusor can be well controlled in the majority of patients, with the result that hyperreflexia is changed into hypo- or areflexia. In most patients treated in this way the residual urine may increase and bladder emptying has then to be assisted or be accomplished by CIC. Contrary to the favourable results with drugs to relax the hyperreflexic detrusor, efficient pharmacological strengthening of the paralysed flaccid striated sphincter musculature is still not possible.

Patients prone to recurrent urinary tract infections which can not be eliminated by other means, require a long term low dosage infection prophylaxis. Recurrent urinary tract infections increase detrusor hyperreflexia by local irritation and, moreover, abolish the effect of the above mentioned spasmolytic drugs.

Intravesical electrotherapy ${ }^{2,3}$ will be mentioned briefly. It is based on the activation of the specific receptors in the bladder wall as confirmed recently by animal experiments. $^{4}$ Depolarisation of these specific receptors by intravesical electrostimulation is the prerequisite for adequate afferent stimuli to induce bladder sensation which in turn is the basis for bladder rehabilitation leading to conscious control of micturition. Therefore patients with incomplete lesions (the usual findings in patients with spinal dysrhaphism) are suitable for this treatment.

Regular bladder emptying, assisted or accomplished by clean intermittent catheterisation, controlled fluid intake and sterile urine are the basis for successful conservative therapy for neurogenic urinary incontinence.

The success rate of various therapeutic strategies for neurogenic urinary incontinence, as presented later, depends on the underlying pathophysiology: both, the detrusor and the sphincter are in the main affected by the neurogenic lesion, either in the same or in a different way (vide infra). To choose the appropriate treatment one has to know whether and to what degree detrusor and sphincter dysfunction contribute to urinary incontinence, and whether a certain dysfunctional pattern, eg hyporeflexia of the detrusor or spasticity of the sphincter, may be useful within a therapeutic concept.

\section{Methods and patients}

One hundred and one children with neurogenic dysfunction of the lower urinary tract, 90 suffering from myelodysplasia, 8 from sacral dysplasia, 1 from a tethered cord syndrome and 2 resulting from an operated dysrhaphic tumour were evaluated. At the time of this investigation all of the patients were over 10 years of age, with a range between 10 and 42 years; all were under the control of our department for at least 2 years.

The results will be presented according to 
the following criteria: continent means dry day and night; socially dry indicates dry intervals for at least 3 hours with regular bladder emptying and controlled fluid intake. If these dry periods are less than 3 hours, these patients were classified as incontinent/wet.

To classify the type of neurogenic lower urinary tract dysfunction a rather simple but functional classification is used, which has been proved to be useful in clinical practice especially as regards therapy, as has previously been described. ${ }^{5}$ A similar classification has already been used by two South American colleagues, Guzman and Windaus. ${ }^{6}$ In this regard there are also the classifications suggested by Krane and Siroky, ${ }^{7}$ by Rickwood et al ${ }^{8}$ and by Mundy et $a l^{9}$ as they draw similar conclusions. Our classification and that of these other authors is based on the fact that in neurogenic lower urinary tract dysfunction, the detrusor and the sphincter may be either hyperreflexic (spastic) or hypo- or areflexic (flaccid). Detrusor and sphincter can be affected likewise - both can either be hyperreflexive or both be hyporeflexive or they may be affected differently; therefore a combination of a hypoflexive detrusor with a hyperreflexive sphincter or vice versa a hyperreflexive detrusor with a hyporeflexive sphincter can be found. Moreover each of these hyper- or hyporeflexive structures can also be combined with a more or less normal counterpart, but these situations are somewhat rare.

\section{Results}

The results will be presented according to the pathophysiology of detrusor and sphincter dysfunction using the above mentioned classification.

\section{Hyperreflexia of the detrusor combined} with hyperreflexia of the pelvic floor musculature response of the striated sphincter

Thirty-four of our young patients, that is, about one third exhibited this type of neurogenic dysfunction. Seven showed the classic detrusor-sphincter dyssynergia, whereas in 27 patients a so called static dyssynergia was present: this means that the sphincter was neither able to contract nor to relax. Out of 34 patients, 14 became continent and 5 socially dry. The overall success rate in this group was $55 \%$.

A further breakdown of the results in this group shows that all patients who empty their bladder regularly by clean intermittent catheterisation became continent or at least socially dry. However, 15 out of 34 are still incontinent; they all empty their bladder, more or less against our advice, mainly using the Credé manoeuvre or by abdominal straining. Intermittent catheterisation was either not accepted or it was tried only for a short time and on an irregular basis. However we can assume that at least some of them would be dry if intermittent catheterisation had been performed properly and over a longer period of time.

Twenty patients in this group who showed at least some sensation for pain in the sacral dermatomes, underwent additional intravesical therapy ${ }^{2-4}$ sometime during the years under observation; $14(70 \%)$ became continent or socially dry. This is mainly because $70 \%$ of the children suitable for this type of treatment do gain at least some form of bladder sensation for the ability to perceive the urge to void.

Five boys, who underwent a sphincterotomy between 8 months and 14 years of age, remained incontinent and are candidates for operative incontinence treatment.

\section{Hyperreflexia of the detrusor combined with hypo-or areflexia of the pelvic floor musculature response of the striated sphincter}

Nineteen patients belong to this group, only 1 achieved continence and 2 became socially dry. In the other 16 conservative treatment was unsuccessful; 5 out of this group received an artificial hydraulic sphincter (Scott). In these patients detrusor hyperreflexia was either controlled by pharmacotherapy or with bladder augmentation performed simultaneously with the implantation of the artificial sphincter. 
Areflexia of the detrusor combined with hyperreflexia of the pelvic floor musculature response of the striated sphincter

There are 11 patients in this group: 4 became continent and 1 socially dry; all empty their bladder by clean intermittent catheterisation; 6 did not achieve urinary continence or the status of being socially dry; and 4 had an artificial hydraulic Scott sphincter implanted. The reason for these somewhat disappointing results is mainly that their sphincters are hyperreflexic by definition but the urethral closure pressure is obviously too low to withstand sudden rises of intraabdominal pressure. According to this, residual urine was not contributing to the situation.

\section{Areflexia of the detrusor combined with areflexia of the pelvic floor musculature response of the striated sphincter}

Of the 32 patients in this group, 23 were treated conservatively: 7 became continent and 5 socially dry with a success rate of $42 \%$. All succeeded in emptying their bladder by CIC, whereas 10 out of 11 who are still incontinent and have not yet undergone a Scott sphincter implantation, empty their bladder by the Credé manoeuvre or abdominal straining.

It is difficult to predict whether and how many of them would have gained continence or the status of being socially dry by performing intermittent catheterisation on a regular basis, because they either did not accept this type of treatment or they performed it only for a short time and/or irregularly. The overall success rate for the 32 patients in this group, including those treated operatively earlier because of their incontinence, was $37 \%$ (12 out of 32 ).

\section{Patients not included in this evaluation}

Three patients now aged 44, 22 and 16 years were not included in these results. They were treated with supravesical diversion at least 15 years ago, because of rapid upper neurogenic urinary tract deterioration. In 2 other patients no urodynamic studies were performed recently, therefore their data were not analysed.

\section{Discussion}

Thirty-four of our patients were classified as having hyperreflexia of the detrusor combined with hyperreflexia of the pelvic floor musculature response of the striated sphincter. Van Gool ${ }^{10}$ found this pattern in $42 \%$ of myelomeningocele patients. With this urodynamic pattern the following conditions increase the chances of conservative therapy enabling a patient to become continent or at least socially dry: (a) a strong spastic striated sphincter which also withstands sudden rises in intraabdominal pressure; (b) pharmacological control of detrusor hyperreflexia or a functional low compliance by spasmolytic medicaments; and (c) bladder emptying by intermittent catheterisation. Only 7 in this group had classic detrusor-sphincter dyssynergia whereas in 27 so called static dyssynergia was present; this means that the sphincter was neither able to contract nor to relax. However, $55 \%$ of this group could achieve continence or the status of being socially dry. The results can be improved by intravesical electrotherapy ${ }^{2-4}$ which is mostly able to induce at least sensation for the urge and the fullness of the bladder in $70 \%$ of the patients suitable for this type of therapy.

With previous transurethral sphincterotomy, the chance of controlling urinary incontinence by conservative therapy is very poor; none of the boys in this group achieved this goal.

If a hyperreflexic detrusor is combined with a hypo- or areflexic sphincter, the chances of success for conservative therapy by whatever means are low, in the range of $10 \%$. The loss of urine during virtually every detrusor contraction and a low bladder volume causes almost constant wetting; CIC brought little change in this pattern.

The best chance of achieving the goal of continence should be if an areflexic detrusor is combined with a hyperreflexic spastic sphincter. We found this type of neuropathic bladder in $11 \%$; Van Gool, in a group of myelodysplastic patients, in $13 \%$. The 
chance of becoming continent or socially dry with this neuropathic bladder type depends totally on the urethral closure pressure, created by the spastic sphincter. Again only those patients who emptied their bladder by CIC became dry.

In the largest group (32\%) urodynamics revealed areflexia of the detrusor combined with areflexia of the pelvic floor musculature response of the striated sphincter. In Van Gool's myelodyplastic patients this urodynamic pattern was present in $35 \% .{ }^{10}$ The success in this group depends mainly on the intraabdominal pressure force and therefore also on the patient's mobility: a wheelchair patient is more prone to stay socially dry than a walker. Such patients usually do not feel or express a sensation of urge or of fullness of the bladder. With CIC the situation can be improved. They may still have stress incontinence, but they need fewer and smaller diapers and manage their incontinence far better socially.

The chances of becoming continent or at least achieving the status of being socially dry by conservative means of treatment depend primarily on the underlying pathophysiology of detrusor and sphincter dysfunction. As in patients with spinal cord injury, urological care of children with myelomeningocele should start on the day of birth. Prevention of secondary damage to the lower and upper urinary tract is a prerequisite for the successful conservative treatment of urinary incontinence. The fact that the majority of children on whom we reported fulfilled these criteria may also explain our favourable results. Moreover we would like to stress that these patients do need further regular urological controls. They should be be monitored closely as regards upper urinary tract function and morphology. Surgical management may become necessary if renal deterioration occurs, even though the patient is continent. Renal damage must not be the price for continence.

If the incontinence situation remains unsatisfactory, a variety of operative procedures can be offered to the patient. Urethral closure can be achieved either by the Scott sphincter or by suspension pro- cedures. ${ }^{11,12}$ Bladder augmentation ${ }^{13}$ is able to abolish the adverse effects of detrusor hyperreflexia and in patients with deteriorated bladders, substitution cystoplasty choosing the natural urethra or a continent diversion for bladder emptying, is performed. Supravesical urinary diversion, frequently performed in the sixties and seventies is nowadays mainly replaced by these procedures. However, all these methods have a considerable percentage of complications and repairs. The long term effects of bladder augmentation or substitution of the bladder with gut segments in this group of young patients cannot yet be predicted. Therefore treatment strategies already in use in early childhood should be directed in such a way that urinary incontinence can be achieved in the future also by conservative treatment.

\section{Conclusions}

The success of conservative therapy in controlling neurogenic urinary incontinence in myelodyplastic children mainly depends on the type of detrusor and sphincter dysfunction and is influenced by the way the bladder is emptied. If detrusor hyperreflexia can be controlled pharmacologically and is combined with a spastic sphincter, the success rate is $55 \%$, provided that the bladder is emptied by intermittent catheterisation. The chances are poor, if a hyperreflexic or poorly compliant bladder is combined with a paralysed flaccid sphincter.

Especially suitable for conservative therapy are patients in whom an areflexic detrusor is combined with a strong spastic sphincter, provided that the bladder is emptied regularly by CIC. An overall success rate of $40 \%$ indicates that a trial of conservative therapy in these patients is worthwhile before any major surgery is considered. Treatment strategies already being used in early childhood should be directed in such a way that a conservative treatment of urinary incontinence may be possible later in life. This should be taken into account especially when considering sphincterotomy. 


\section{References}

1 Gross RE (1953) The Surgery of Infancy and Childhood. Saunders, Philadelphia.

2 Katona F (1974) Stages of vegetative afferentation in reorganisation of bladder control during electrotherapy. Urol Ing 30: 19-26.

3 Madersbacher H (1990) Intravesical electrical stimulation for the rehabilitation of the neuropathic bladder. Paraplegia 28: 349-352.

4 Ebner A, Lindström S, Jiang C-H (1991) Intravesical electrostimulation: how does it work? An experimental study. J Neurourol Urodyn 10: 273-280.

5 Madersbacher H (1990) The various types of neurogenic bladder dysfunction: an update of current therapeutic concepts. Paraplegia 28: 217-229.

6 Guzman JM, C Windaus (1981) Agrupamento urodinamico de los sindromes de vejiga neurogenica. Pediatria 5: $28-33$.

7 Krane JR, Siroky MR (1991) Neurogenic vesicourethral dysfunction. In: JR Krane, MR Siroky, editors. Clinical Neurology. Little Brown \& Co, Boston: 319-344.

8 Rickwood AMK, Thomas DG, Philp NH, Spicer RD (1982) Assessment of congenital neurovesical dysfunction by combined urodynamic and radiological studies. Brit J Urol 54: 512-518.

9 Mundy AR, Shah PJR, Borzykowski M, Saxton HM (1985) Sphincter behaviour in myelomeningocele. Brit J Urol 57: 647-651.

10 Gool JD van (1986) Spina Bifida and Neurogenic Bladder Dysfunction, a Urodynamic Study. Uitgevery Impress, Utrecht.

11 McGuire JE, Chi-Chung Wang, Usitelo H, Savastano J (1986) Modified pubovaginal sling in girls with myelodysplasia. J Urol 135: 94-99.

12 Elder JS (1992) Puboprostic sling in boys with continence. J Urol 147: 252.

13 Stephenson TP, Mundy AR (1985): Treatment of the neuropathic bladder by enterocystoplasty and selective sphincterotomy or sphincter ablation and replacement. Brit J Urol 57: 27-31. 